\title{
Indirect calorimetry: a tool to adjust energy expenditure in very low birth weight infants
}

\author{
Calorimetria indireta: uma ferramenta para adequação das necessidades \\ energéticas dos recém-nascidos de muito baixo peso ao nascer
}

\section{Fernanda V. M. Soares ${ }^{1}$, Maria E. L. Moreira², Andrea D. Abranches ${ }^{3}$, José R. M. Ramos ${ }^{4}$,} Saint C. S. Gomes Junior ${ }^{5}$

\section{Resumo}

Objetivo: Verificar o gasto energético de repouso dos recémnascidos de muito baixo peso ao nascer através da calorimetria indireta.

Métodos: Estudo transversal com 29 recém-nascidos de muito baixo peso ao nascer, clinicamente estáveis, recebendo dieta $>100$ $\mathrm{kcal} / \mathrm{kg} / \mathrm{dia}$. A desnutrição foi determinada como todo escore $z$ do peso para idade $\leq-2 \mathrm{DP}$. A aferição do gasto energético de repouso foi realizada através da calorimetria indireta com o sistema aberto.

Resultados: No momento do exame, o peso médio foi de $1.564 \pm 393 \mathrm{~g}$, idade corrigida de $35 \pm 3$ semanas, e a desnutrição estava presente em $62,1 \%$. O valor médio da taxa metabólica basal foi de $57,01 \pm 7,76 \mathrm{kcal} / \mathrm{kg} / \mathrm{dia}$, maior do que o usado como referência.

Conclusão: $O$ elevado gasto energético de repouso pode estar exercendo um forte impacto no desenvolvimento da desnutrição no período de hospitalização, pois o gasto energético de repouso é o principal componente calórico do gasto energético total.

J Pediatr (Rio J). 2007;83(6):567-570: Nutrição, calorimetria indireta, pré-termos.

\section{Introdução}

Nutrir adequadamente o recém-nascido (RN) prematuro com o objetivo de proporcionar-lhe um crescimento extraútero semelhante ao crescimento fetal é um dos grandes desafios da neonatologia. Apesar da importância do manuseio nutricional dos RN prematuros estar claramente reconhecida, as quantidades necessárias da maioria dos nutrientes

\section{Abstract}

Objective: To determine the resting metabolic rate in very low birth weight infants through indirect calorimetry.

Methods: Cross-sectional study including 29 clinically stable very low birth weight infants receiving an enteral diet $>100$ $\mathrm{kcal} / \mathrm{kg} /$ day. Malnutrition was defined as weight-for-age $\mathrm{z}$ score $\leq-$ $2 \mathrm{SD}$. Resting energy expenditure was measured using open circuit indirect calorimetry.

Results: At the time of the examination, mean weight was $1,564 \pm 393 \mathrm{~g}$ and corrected gestational age was $35 \pm 3$ weeks. Malnutrition was diagnosed in $62.1 \%$ of the preterm infants. The mean resting metabolic rate was $57.01 \pm 7.76 \mathrm{kcal} / \mathrm{kg} / \mathrm{day}$.

Conclusion: Since resting energy expenditure is the main caloric component of total energy expenditure, the high resting metabolic rate observed may have a strong impact on the development of malnutrition during hospitalization.

J Pediatr (Rio J). 2007;83(6):567-570: Nutrition, indirect calorimetry, preterm infant.

não estão bem estabelecidas. Estas incertezas podem contribuir para ingestão limitada de nutrientes, e sabe-se que uma nutrição inadequada em períodos precoces da vida pode apresentar um impacto no desenvolvimento a longo prazo ${ }^{1}$.

O objetivo principal da nutrição neonatal é proporcionar uma velocidade de crescimento semelhante ao intra-útero, portanto fatores como metabolismo basal, atividade física,

1. Nutricionista. Mestranda, Instituto Fernandes Figueira, Fundação Oswaldo Cruz, Rio de Janeiro, RJ.

2. Doutora. Médica. Docente permanente, Pós-Graduação em Saúde da Criança e da Mulher, Instituto Fernandes Figueira, Fundação Oswaldo Cruz, Rio de Janeiro, RJ.

3. Nutricionista. Bolsista de pesquisa, Convênio Fundação Oswaldo Cruz (Fundação Carlos Chagas Filho de Amparo à Pesquisa do Estado do Rio de Janeiro FAPERJ), Rio de Janeiro, RJ.

4. Doutor. Médico. Docente, Programa de Pós-Graduação, Instituto Fernandes Figueira, Fundação Oswaldo Cruz, Rio de Janeiro, RJ.

5. Doutor. Pesquisador, Instituto Fernandes Figueira, Fundação Oswaldo Cruz, Rio de Janeiro, RJ. Estatístico.

Fonte financiadora: DECIT(MS)/CNPq.

Não foram declarados conflitos de interesse associados à publicação deste artigo.

Como citar este artigo: Soares FV, Moreira ME, Abranches AD, Ramos JR, Gomes Junior SC. Indirect calorimetry: a tool to adjust energy expenditure in very low birth weight infants. J Pediatr (Rio J). 2007;83(6):567-570.

Artigo submetido em 13.03.07, aceito em 11.07.07.

doi:10.2223/JPED. 1719 
manutenção térmica, digestão, síntese e perdas devem ser levados em consideração ao se prescrever a alimentação para o RN².

Existem vários métodos para mensurar o gasto energético, como calorimetria direta, calorimetria indireta, água duplamente marcada, princípio de Fick. Dentre estes, destaca-se a calorimetria indireta, que é um método prático, seguro, não-invasivo e portátil que mede o gasto energético a partir do $\mathrm{VO}_{2}$ e da produção de gás carbônico obtidos pela análise do ar inspirado e expirado pelos pulmões. Além disso, a calorimetria indireta é considerada o método mais adequado e seguro para ser aplicado em RN, além de poder ser usada para medir longos períodos de tempo ${ }^{3}$.

O objetivo deste estudo foi verificar o gasto energético de repouso (GER) dos RN de muito baixo peso ao nascer através do exame de calorimetria indireta.

\section{Métodos}

\section{Caracterização da população}

Foi realizado um estudo transversal em um grupo de RN admitidos na unidade de terapia intensiva neonatal do Instituto Fernandes Figueira, Rio de Janeiro, com peso de nascimento inferior a $1.500 \mathrm{~g}$, internados no período de 6 meses (junho a dezembro de 2005). Foram excluídos do estudo os RN que apresentavam malformação congênita, infecção congênita, hemorragia intracraniana grau III e IV e sepse. O tamanho amostral para estimar o GER foi determinado em 29 RN, considerando uma significância de $95 \%$ e poder de $80 \%$. As estimativas para o cálculo da amostra foram obtidas dos estudos usando o mesmo método ${ }^{4}$. Todos os RN internados no período do estudo que obedecessem ao critério de inclusão e cujas mães autorizassem foram admitidos no estudo, consecutivamente, até atingir o tamanho amostral.

A avaliação das medidas foi realizada nos RN internados após o $14^{\circ}$ dia de vida e a partir do momento em que estes estivessem clinicamente estáveis, em ar ambiente e recebendo exclusivamente dieta enteral superior a $100 \mathrm{kcal} / \mathrm{kg} /$ $\mathrm{dia}^{4}$. Todos os RN estavam em fase de crescimento, já haviam recuperado o peso de nascimento e 81,3 \% deles estavam em uso de dieta mista (leite humano fortificado e fórmula). Nenhum deles estava usando medicamentos (aminofilina, cafeína ou digital, anticonvulsivantes) ou apresentavam patologias (sepse, displasia broncopulmonar) que pudessem interferir no gasto metabólico. A idade gestacional ao nascimento foi obtida pela data da última menstruação, confirmada pela ultra-sonografia de primeiro trimestre e, em caso de dúvida, pelo método de Ballard et al. ${ }^{5}$.

$\mathrm{Na}$ avaliação da adequação nutricional no momento do exame, foi usado o escore $z$ do peso para idade, utilizando como referência os dados de distribuição de peso para idade gestacional canadense ${ }^{6}$. A desnutrição foi determinada como todo escore $z$ do peso para idade $\leq-2$ (próximo ao percentil 3), limite estabelecido pela Organização Mundial da Saúde $(\mathrm{OMS})^{7}$.

\section{Avaliação metabólica}

A aferição do $\mathrm{GER}$, consumo de oxigênio $\left(\mathrm{VO}_{2}\right)$, produção de dióxido de carbono $\left(\mathrm{VCO}_{2}\right)$ e coeficiente respiratório (RQ) foi realizada através da calorimetria indireta, com o uso do Deltatrac II Metabolic Monitor. Este equipamento usa o princípio do circuito aberto, que permite a medição contínua do $\mathrm{VO}_{2}$ e $\mathrm{VCO}_{2}$ usando um gerador de fluxo contínuo. As medidas de calorimetria indireta foram iniciadas 1 hora após a dieta, e o tempo de realização do exame foi de 20 minutos após estabilização do aparelho ${ }^{8}$. As medidas foram obtidas através de uma campânula colocada ao redor da face do RN, que foi mantido quieto, em posição confortável e na incubadora com zona termoneutra, conforme tabela de Scopes \& Ahmed $^{9}$. A temperatura foi monitorizada pelo servocontrole adaptado à pele do RN. O exame foi interrompido quando o bebê se apresentava acordado ativo ou chorando, seguindo uma escala que faz uma associação entre atividade corporal e gasto energético ${ }^{10}$.

\section{Análise estatística}

Foi utilizado um software estatístico para criação do banco de dados e análise das variáveis. As variáveis foram descritas através de medidas de freqüência, médias e seus respectivos desvios padrão.

Este estudo foi aprovado pelo Comitê de Ética em Pesquisa com Seres Humanos do Instituto Fernandes Figueira e o termo de consentimento livre esclarecido foi devidamente autorizado pelos responsáveis.

\section{Resultados}

Foram estudados 29 RN com peso de nascimento médio de $1.210 \pm 231 \mathrm{~g}$ e idade gestacional de $31 \pm 3$ semanas ao nascimento. As características da população estudada ao nascimento e no momento do exame estão descritas na Tabela 1.

Em relação ao estado nutricional, verificamos que houve um aumento significativo ( 21 para $62,1 \%$ ) do número de RN abaixo do escore $z$ do peso para idade $\leq-2,0$ entre o nascimento e o momento da avaliação.

O GER da população estudada aferida pela calorimetria

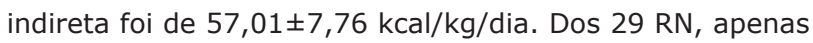
seis $(20,6 \%)$ apresentavam um GER entre 40 e $50 \mathrm{kcal} / \mathrm{kg} /$ dia, valor utilizado como referência pela Academia Americana de Pediatria (AAP).

\section{Discussão}

Os RN de muito baixo peso ao nascer constituem um grupo de risco nutricional, portanto estudos relacionados à nutrição e seu impacto no crescimento são tão importantes e atuais. 0 ganho de peso insuficiente pode ser um importante marcador de nutrição inadequada, o que, por sua vez, tem um grande impacto no desenvolvimento cognitivo, com diminuição de células cerebrais ${ }^{11}$.

Para a AAP, a qualidade do ganho de peso pós-natal depende do tipo, quantidade e qualidade da dieta oferecida ${ }^{2}$. 
Tabela 1 - Características dos recém-nascidos de muito baixo peso ao nascer internados na unidade de terapia intensiva neonatal no nascimento e no momento do exame de calorimetria indireta

\begin{tabular}{|c|c|}
\hline Variáveis & Média \pm desvio padrão \\
\hline \multicolumn{2}{|l|}{ Ao nascimento } \\
\hline Peso $(g)^{*}$ & $1.210 \pm 231$ \\
\hline Idade gestacional (semanas)* & $31 \pm 3$ \\
\hline Escore $z^{*}$ & $-1,3 \pm 1,2$ \\
\hline Desnutridos, n (\%) & $7(21 \%)$ \\
\hline \multicolumn{2}{|l|}{ Ao exame } \\
\hline Peso $(\mathrm{g})^{*}$ & $1.564 \pm 393$ \\
\hline Idade corrigida (semanas)* & $35 \pm 3$ \\
\hline Escore $z^{*}$ & $-2,1 \pm 1,5$ \\
\hline Desnutridos, n (\%) & $22(62,1 \%)$ \\
\hline \multicolumn{2}{|l|}{ Calorimetria indireta } \\
\hline Gasto energético de repouso ( $\mathrm{kcal} / \mathrm{kg} / \mathrm{dia})^{*}$ & $57,01 \pm 7,76$ \\
\hline $\mathrm{VCO}_{2}(\mathrm{~mL} / \mathrm{kg} / \mathrm{min})^{*}$ & $6,74 \pm 2,33$ \\
\hline $\mathrm{VO}_{2}(\mathrm{~mL} / \mathrm{kg} / \mathrm{min})^{*}$ & $7,33 \pm 2,56$ \\
\hline $\mathrm{RQ}^{*}$ & $0,92 \pm 0,08$ \\
\hline
\end{tabular}

* Média \pm desvio padrão.

$\mathrm{RQ}=$ coeficiente respiratório; $\mathrm{VCO}_{2}=$ produção de dióxido de carbono; $\mathrm{VO}_{2}=$ consumo de oxigênio.

Temos observado que, em nosso meio, a incidência de desnutrição dos RN prematuros ao atingirem a idade corrigida do termo chega a $63 \%{ }^{12}$. Muitos fatores podem estar contribuindo para um ganho inadequado de peso: estimativas incorretas da taxa metabólica, oferta insuficiente e absorção inadequada, com a taxa de excreção de nutrientes alta.

Em pacientes como os prematuros extremos, o uso da calorimetria indireta pode fornecer subsídios importantes para o manuseio nutricional, pois o dispêndio energético desses pacientes é muito variável. As estimativas baseadas somente em equações preditivas genéricas podem subestimar suas reais necessidades, prejudicando o crescimento e desenvolvimento e, conseqüentemente, a qualidade de vida desses bebês ${ }^{3}$.

A AAP atribui para os RN prematuros de baixo peso um GER variando de 40 a $50 \mathrm{kcal} / \mathrm{kg} / \mathrm{dia}^{2}$. Estudos com calorimetria indireta têm sido utilizados para verificar o GER dos RN, sendo de particular importância, pois a magnitude da alteração do gasto energético pode ter conseqüências no crescimento a longo prazo ${ }^{13}$. Bauer et al. ${ }^{4}$, estudando RN prematuros com idade gestacional inferior a 30 semanas, encontraram um GER de $53 \pm 6 \mathrm{kcal} / \mathrm{kg} / \mathrm{dia}$ no $21^{\circ}$ dia de vida. Já Putet et al. ${ }^{14}$ avaliaram o GER de RN de muito baixo peso ao nascer ao completarem 36 semanas de idade corrigida e verificaram um gasto energético de $51,5 \pm 2,9 \mathrm{kcal} / \mathrm{kg} / \mathrm{dia}$, quando alimentados por pool de leite humano pasteurizado, e de $63,3 \pm 4,5 \mathrm{kcal} / \mathrm{kg} / \mathrm{dia}$, quando alimentados por fórmula específica para prematuros. Os resultados encontrados em nosso estudo $(57,01 \pm 7,76 \mathrm{kcal} / \mathrm{kg} / \mathrm{dia})$ assemelham-se aos resultados dos estudos acima citados, que também estão acima do sugerido pela $A A^{2}$, evidenciando uma necessidade de revisão da oferta calórica recomendada, uma vez que os RN de muito baixo peso podem estar gastando mais energia para manutenção dos processos vitais e, conseqüentemente, aumentando a sua necessidade calórica. Esses resultados podem estar refletindo no ganho de peso inadequado encontrado em nosso estudo.

O gasto energético basal é responsável por manter os processos vitais do organismo em condições controladas e padronizadas, com o indivíduo em repouso e com a temperatura ambiente adequada. $\mathrm{O}$ gasto energético em repouso corresponde ao gasto energético nas mesmas condições do gasto energético basal, porém pode ser medido após refeição ou atividade normal. Em RN e lactentes, o GER assemelha-se ao gasto energético basal ${ }^{15}$.

O elevado GER pode estar exercendo um forte impacto no desenvolvimento da desnutrição no período de hospitalização, pois o gasto energético basal é o principal componente calórico do gasto energético total e fundamental para uma adequada prescrição calórica.

A influência no gasto energético de fatores como condição clínica do RN, tipo de leite, uso de drogas com xantinas, diuréticos, entre outros, ainda precisa ser avaliada através de estudos específicos para estes objetivos, já que o desenho 
e o tamanho amostral deste estudo exploratório não estão adequados para estes objetivos.

Estudos como este, usando a técnica da calorimetria indireta, são importantes nessa faixa etária, porque deles podem-se originar revisões das necessidades nutricionais dos prematuros no sentido de proporcionar-lhes crescimento e desenvolvimento adequados.

\section{Agradecimento}

À nutricionista Priscila Panisset de Figueredo, pela colaboração na coleta dos dados.

\section{Referências}

1. Hay WW. Early postnatal nutritional requirements of the very preterm infant based on a presentation at the NICHD-AAP workshop on research in neonatology.J Perinatol. 2006;26 Suppl 2:S13-8.

2. American Academy of Pediatrics, Commitee on Nutrition. Nutrition needs of preterm infants. In: Kleinman RE, editor. Pediatric Nutrition Handbook. 5th ed. Elk Grove, IL: American Academy of Pediatrics; 2004. p. 36.

3. Kashyap S, Schulze KF. Energy requeriments and protein-energy metabolism and balance in preterm and term infants. In: Thureen PJ, Hay WW, editors. Neonatal nutrition and metabolism. Cambridge: Cambridge University; 2006. p. 134-46.

4. Bauer K, Laurenz M, Ketteler J, Versmold H. Longitudinal study of energy expenditure in preterm neonates $<30$ weeks' gestation during the first three postnatal weeks.J Pediatr.2003;142:390-6.

5. Ballard JL, Khoury JC, Wedig K, Wang L, Eilers-Walsman BL, Lipp R. New Ballard score, expanded to include extremely premature infants.J Pediatr.1991;119:417-23.

6. Kramer MS, Platt RW, Wen SW, Joseph KS, Allen A, Abrahamowicz $M$, et al. A new and improved population-based Canadian reference for birth weight for gestational age. Pediatrics. 2001;108:E35.
7. World Health Organization (WHO) Expert Commitee on Physical Status. Physical status: the use and interpretation of anthropometry. Geneve: WHO; 1995. WHO Technical Report Series 854 .

8. Moreira ME, Vieira AA, Mendes Soares FV, Bastos Lopes R, Gomes $P$, Abranches $A D$, et al. Determining the least time required for measuring energy expenditure in premature neonates.J Perinat Med.2007;35:71-5.

9. Scopes JW, Ahmed I. Minimal rates of oxygen consumption in sick and premature newborn infants. Arch Dis Child.1966; 41:407-19.

10. Thureen PJ, Phillips RE., Baron KA, De Marie MP, Hay WW. Direct measurement of the energy expenditure of physical activity in preterm infants. J Appl Physiol. 1998;85:223-30.

11. Ziegler EE. Breast milk fortification.Acta Paediatr.2001; 90:720-3.

12. Gianini NM, Vieira AA, Moreira ME. Avaliação dos fatores associados ao estado nutricional na idade corrigida de termo em recém-nascidos de muito baixo peso.J Pediatr (Rio J).2005; 81:34-40.

13. Thureen PJ. Measuring energy expenditure in preterm and unstable infants.J Pediatr.2003;142:366-7.

14. Putet G, Senterre J, Rigo J, Salle B. Nutrient balance, energy utilization, and composition of weight gain in very-low-birthweight infants fed pooled human milk or a preterm formula.J Pediatr.1984;105:79-85.

15. Food and Agriculture Organization (FAO)/World Health Organization (WHO)/United Nations University (UNU) Expert Consultation. Energy and protein requirements. Geneva: WHO; 1985. WHO Technical Report Series 724. http://www.fao.org/ DOCREP/003/AA040E/AA040E04.htm Acesso: 10/02/2007.

Correspondência:

Maria Elisabeth Lopes Moreira

Instituto Fernandes Figueira - Departamento de Neonatologia

Av. Rui Barbosa 716, Flamengo

CEP 22250-020 - Rio de Janeiro, RJ

Tel.: (21) 2554.1819

E-mail: bebeth@iff.fiocruz.br 